\title{
Genomic characterization of the inflammatory response initiated by surgical intervention and the effect of perioperative cyclooxygenase 2 blockade
}

\author{
Keith D. Coon, $\mathrm{PhD},{ }^{\mathrm{a}}$ Landon J. Inge, $\mathrm{PhD},{ }^{\mathrm{a}}$ Kristen Swetel, ${ }^{\mathrm{a}}$ Valerie Felton, ${ }^{\mathrm{a}}$ Phillip Stafford, $\mathrm{PhD},{ }^{\mathrm{b}}$ and \\ Ross M. Bremner, MD, PhD
}

Objective: There are potentially deleterious sequelae of the physiologic response to surgical intervention. Some inflammatory cytokines can act as tumor growth factors, angiogenic and metastatic promoters, or both. Modulation of negative effects could improve outcomes from surgical intervention. The effects of surgical intervention on gene expression have not been fully elucidated. We assayed gene expression changes in an animal model of thoracotomy versus a sham operation and evaluated the ability of a cyclooxygenase inhibitor, celecoxib, to mediate these changes.

Methods: Sixty adult male BALB/c mice were randomized into one of 3 experimental groups: sham operation with anesthesia only, thoracotomy incision, and thoracotomy incision with perioperative celecoxib administration. Six hours after surgical intervention, the animals were killed, and blood was collected. RNA pools from each group were labeled and hybridized to Mouse Whole Genome Microarrays. Gene expression profiles were analyzed to determine the effect of both surgical intervention and celecoxib treatment.

Results: Surgical intervention initiated a robust gene expression response. We identified 867 transcripts that were found to be statistically significant (corrected $P<.05$ ) and differentially expressed at least 2-fold in response to surgical intervention. Celecoxib had a profound effect on this response, preserving close to baseline levels of expression for most of those genes.

Conclusions: Surgical intervention has a dramatic effect on the expression of genes related to the inflammatory response. Perioperative treatment with a cyclooxygenase 2 inhibitor abated many of these changes and might counteract many of the negative effects of the response to surgical intervention. (J Thorac Cardiovasc Surg 2010;139:1253-60)

Supplemental material is available online.

Surgical procedures elicit trauma-related changes in what is known as the "surgical stress response." Typically, surgical intervention induces activation of immune cells and subsequent release of various cytokines, which have farreaching systemic effects. Initially, cytokines are produced by monocytes and macrophages at the local site of injury, ${ }^{1}$ but this is followed by participation of peripherally recruited leukocytes. This cytokine cascade plays a significant role in the resulting acute-phase immune response, ${ }^{2}$ which typically

\footnotetext{
From the Center for Thoracic Disease, ${ }^{\text {a }}$ Heart and Lung Institute, St. Joseph's Hospital and Medical Center, Phoenix, Ariz; and the Center for Innovations in Medicine, ${ }^{b}$ Biodesign Institute, Arizona State University, Tempe, Ariz.

Disclosures: None.

Read at the Thirty-fifth Annual Meeting of The Western Thoracic Surgical Association, Banff, Alberta, Canada, June 24-27, 2009.

Received for publication June 20, 2009; revisions received Dec 16, 2009; accepted for publication Jan 18, 2010.

Address for reprints: Ross M. Bremner, MD, PhD, 500 W Thomas Rd, Suite 500,

Phoenix, AZ 85013 (E-mail: ross.bremner@chw.edu).

$0022-5223 / \$ 36.00$

Copyright (C) 2010 by The American Association for Thoracic Surgery

doi:10.1016/j.jtcvs.2010.01.022
}

normalizes within a few days of the surgical injury, as determined based on measurement of inflammatory mediators. ${ }^{3,4}$

Cytokines mediate cell signaling through initiation, amplification, and perpetuation of the inflammatory response at the local and systemic levels. Many of these signals are proinflammatory. Several attempts have been made to illustrate the effect of surgical intervention on the inflammatory response in peripheral blood using cytokines, either in serum $^{3-5}$ or by assessment of cytokine mRNA in blood monocytes. ${ }^{6}$ However, precise characterization of this response, particularly at the molecular level, has been limited because of the small number of markers examined and largely inconclusive results. The outcomes of these experiments vary by the temporal specificity of peak cytokine expression, ${ }^{3-5,7}$ the magnitude of the surgical procedure, ${ }^{3,5,8}$ and the tissue in which the response was assessed. ${ }^{9}$

Both anesthesia and the act of surgical intervention itself result in significant immunosuppression, ${ }^{10}$ which can have important consequences for surgical resection of cancers. Much of the inflammatory response at the time of surgical intervention has been suggested to have a negative effect in terms of metastasis and tumor recurrence. ${ }^{8,11}$ Furthermore, surgeons have historically not used systemic anticancer therapies during the perioperative period for fear of interfering with a successful surgical outcome. The 


\section{Abbreviations and Acronyms \\ COX-2 = cyclooxygenase 2 \\ IFN- $\gamma=$ interferon $\gamma$ \\ $\mathrm{NK}=$ natural killer}

immune suppression of surgical intervention and anesthesia coupled with the lack of any systemic therapy in the perioperative period leaves the patient vulnerable to the establishment of micrometastatic deposits or to growth of established metastases. Indeed, it has been suggested that this vulnerability could also activate an angiogenic switch for quiescent metastases. The perioperative administration of an agent that could alleviate the negative impact of surgical intervention and provide some systemic therapeutic oversight has the potential to improve survival after surgical intervention. One promising group of compounds with the potential for use at the time of surgical intervention is the cyclooxygenase 2 (COX-2) inhibitors. The COX-2 enzyme is upregulated by surgical stress and appears to modulate many of the inflammatory pathways after surgical intervention. It is also known to occur in many cancers, including non-small cell lung cancer. ${ }^{12}$ The COX-2 inhibitor, celecoxib, was developed to provide anti-inflammatory and analgesic benefits to patients with severe arthritic pain. It has little effect on bleeding or renal dysfunction and does not appear to have significant cardiovascular side effects, especially when used for limited periods of time. ${ }^{13}$ It also demonstrates profound anticancer effects through mechanisms that involve apoptosis and the inhibition of angiogenesis and metastasis. ${ }^{14}$

The aims of this experiment were to (1) more precisely characterize the systemic inflammatory response to surgical intervention at the genomic level and (2) determine the potential for pharmacologic modulation of that response using a compound, celecoxib, known to have both antiinflammatory and anticancer benefits. To achieve these goals, we performed whole-genome gene expression microarray analysis on mice that had been subjected to anesthesia alone (group A), anesthesia and a surgical procedure (group AS), and anesthesia and a surgical procedure with the addition of perioperative administration of celecoxib (group ACS). Gene expression analysis quantifies the activity of a gene by measuring the number of transcripts being produced under a given experimental condition, and the use of microarrays permits the evaluation of all known genes in a single experiment, making the identification of inflammatory pathways substantially more robust than has previously been possible. Our goal was to elucidate potentially deleterious aspects of the response to surgical intervention and determine whether we could modulate those effects through the use of a COX-2 inhibitor known to have antitumor activity to ascertain its potential clinical utility.

\section{MATERIALS AND METHODS}

\section{Animals and Treatments}

Sixty 5- to 6-week-old male BALB/cAnNTac mice were obtained from Taconic Farms, Inc (Germantown, NY), and acclimated for 8 days. The mice were housed 4 per cage in Thoren polycarbonate maxi-miser cages and racks (Lithgow Laboratory Services, Las Vegas, Nev) with Aspen Sani-chips (Harlan Teklan, Madison, Wis). They were fed Purina Lab diet for rodents and given sterile water for irrigation. The animal rooms were maintained at $20^{\circ} \mathrm{C}$ to $23^{\circ} \mathrm{C}$ and $48 \%$ to $53 \%$ relative humidity with a 12/12-hour light/dark cycle. Mice were randomized into 3 groups of 20. Group A underwent a "sham" operation (anesthesia only). Group AS underwent a thoracotomy incision, a procedure that mimics the surgical stress of an actual thoracotomy without opening the pleural cavity, thus preventing pneumothorax. Group ACS was administered perioperative celecoxib (lot no. C070030; G.D. Searl LLC, New York, NY) treatment (100 $\mathrm{mg} / \mathrm{kg}$ by weight per day by means of oral gavage) for 3 days, followed by a thoracotomy incision. All surgical procedures were performed after achievement of anesthesia (through intraperitoneal injection with ketamine/xylazine) by a board-certified thoracic surgeon. Anesthesia protocols for all groups were consistent. Six hours after surgical intervention, anesthesia was readministered, and cardiac puncture was performed to collect blood. The 6-hour postoperative time point was chosen based on available data for assessing the systemic response to injury. ${ }^{3-8,11}$

\section{Isolation of High-Quality RNA}

Blood was immediately processed for isolation of total RNA by using the Mouse RiboPure Blood RNA Isolation Kit (Ambion, Austin, Tex), according to the manufacturer's specifications. The 60 individual RNAs were quantified in triplicate by using the Quant-it RNA Assay Kit and Qubit flourometer (Invitrogen, Carlsbad, Calif), according to the manufacturer's specifications. Absorbance at 260/280 and 260/230 was measured to assess RNA quality, and the best 16 RNA samples from each group were pooled in equimolar concentrations to create a single RNA pool per group. Each of the 3 resulting RNA pools was diluted to $50 \mathrm{ng} / \mu \mathrm{L}$ and assessed on an Agilent 2100 bioanalyzer (Agilent, Foster City, Calif) to ensure only RNA of the highest integrity was used for subsequent microarray analysis.

\section{Microarray Analysis}

Each of the 3 RNA pools was labeled and hybridized (in quadruplicate) to Agilent 4x44K One-Color Whole Mouse Genome Oligo Microarrays, scanned on an Agilent Microarray Scanner (model G2505B), and processed with Agilent's Feature Extraction software (version 9.5.1). All arrays were quality controlled (ie, normalized) for a minimum median hybridization intensity with GeneSpring 10.0.2 software (Agilent). One of the 4 technical replicates for group 3 displayed significant amounts of error and was removed from further analysis. Expression of each gene was averaged across technical replicates. Unpaired $t$ tests were used to determine gene expression differences between groups by using the false discovery rate multiple testing correction. ${ }^{15}$ Statistically significant entities were assessed for a minimum 2 -fold change in GeneSpring version 10.0.2. Because multiple probes can be used to assess the presence or absence of individual genes on a microarray, data will be described in both/either terms of transcripts (ie, probes), genes, or both, depending on the context. A flowchart of the experimental design for this investigation can be seen in Figure 1.

\section{RESULTS}

\section{Patterns of Gene Expression Associated With Surgical Intervention and Celecoxib Administration}

High-quality, robust gene expression data were obtained for all 3 experimental groups at the chosen 6-hour time point, and a total of 948 differentially expressed transcripts were 


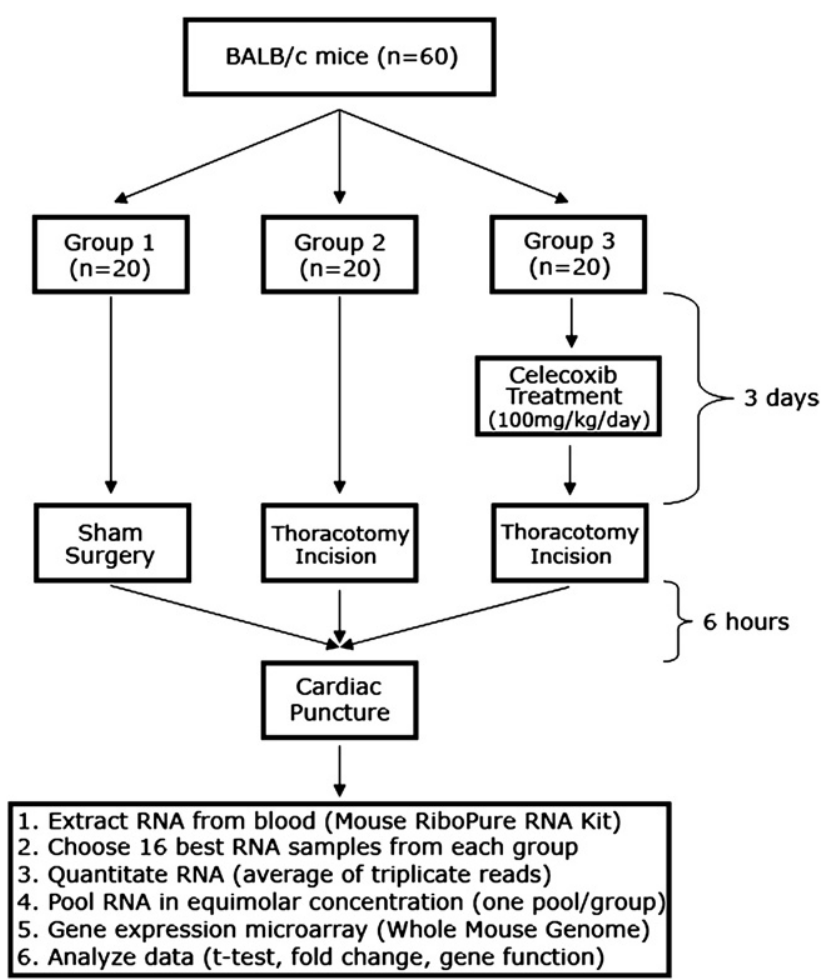

FIGURE 1. A flowchart of the experimental design outlined in this study.

identified at a $99 \%$ confidence interval. Comparison of the surgical intervention group (group AS) with the baseline group (group A) identified 867 transcripts ( 770 genes) that were found to be statistically significant (corrected $P<.05)$ and differentially expressed at least 2 -fold. The vast majority of these transcripts (769) were upregulated in response to surgical intervention, with only 98 being downregulated. Perioperative administration of celecoxib had a dramatic effect on gene expression changes induced by surgical intervention. Contrast of the expression pattern of the 867 transcripts differentially expressed in the surgical intervention group to that of the same 867 transcripts in the celecoxib-treated group can be seen in Figure 2. The surgically induced increase in gene expression (Figure 2, A) was significantly modulated by the perioperative administration of the COX-2 inhibitor (Figure 2, B).

Comparison of the celecoxib-treated surgical intervention group (group ACS) with the baseline group (group A) identified 216 transcripts (190 genes) that were found to be statistically significant (corrected $P<.05$ ) and differentially expressed at least 2-fold, of which 199 were upregulated and 17 were downregulated. A Venn diagram showing the relationship between our 2 experimental groups can be seen in Figure 3. This analysis revealed 3 distinct sets of genes: those that are differentially expressed only in response to surgical intervention alone (732 transcripts), those that are differentially expressed in response to both surgical intervention and celecoxib treatment (135 transcripts), and those that, by inference, are differentially expressed in response to celecoxib treatment alone (81 transcripts). A 2-dimensional unsupervised hierarchical clustering was performed on the 948 differentially expressed transcripts
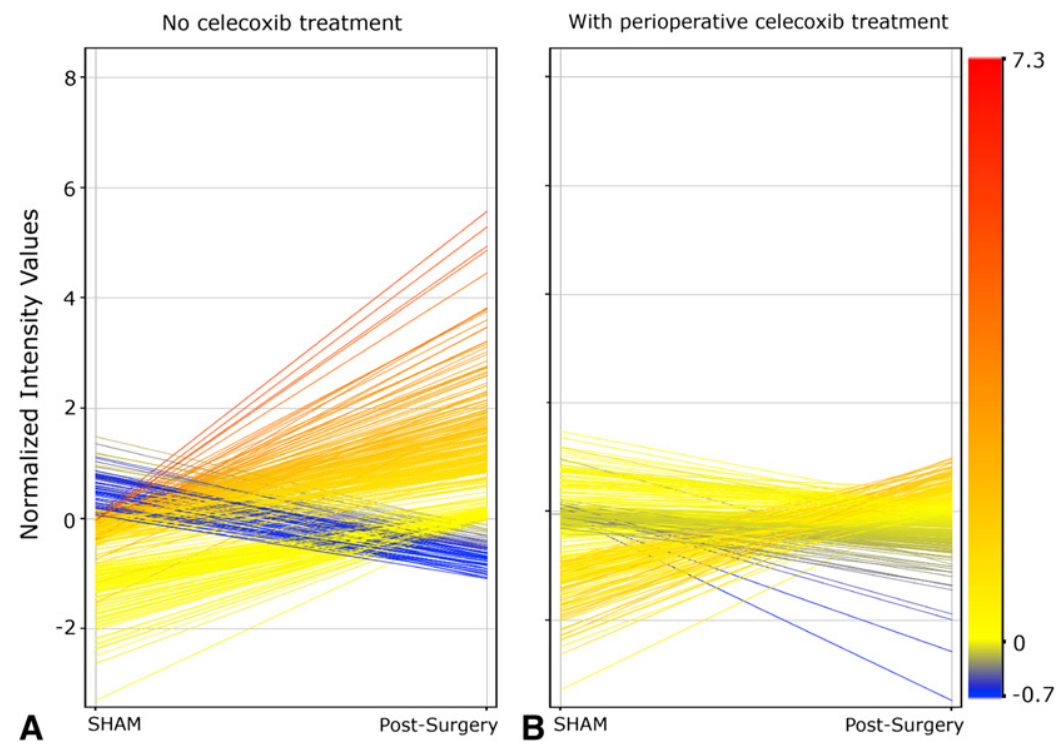

FIGURE 2. Expression profile showing the normalized intensity values of 867 transcripts ( 770 genes) found to be statistically significant (corrected $P<.05$ ) and differentially expressed at least 2-fold at 6 hours after surgical intervention in a group of mice subjected to anesthesia followed by a surgical procedure (Post-Surgery) compared with a group of mice subjected to anesthesia only (SHAM). A, No perioperative treatment. B, With perioperative administration of the COX-2 inhibitor celecoxib. The colored lines in these profile plots indicate the direction of gene regulation (up or down), with increases in gene expression displayed in the orange-to-red spectrum, decreases in gene expression displayed in the green-to-blue spectrum, and marginal gene expression changes displayed in yellow. 


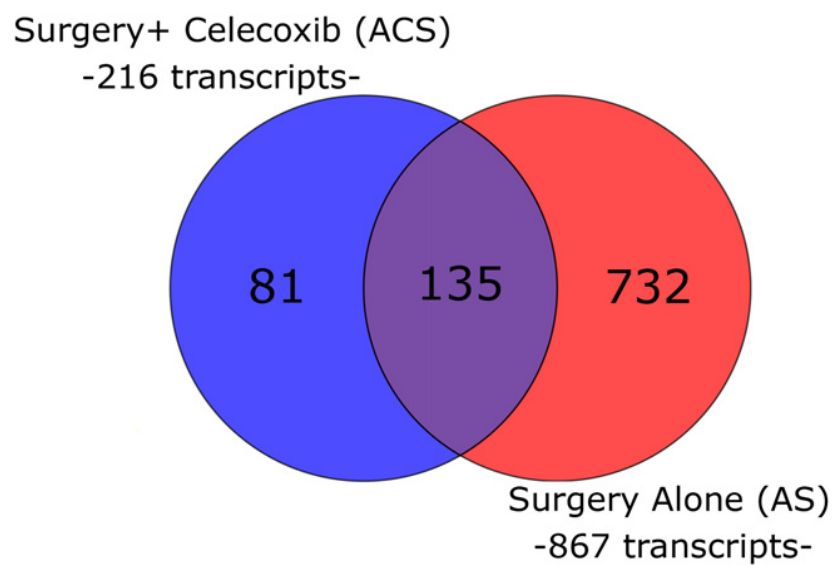

FIGURE 3. A Venn diagram showing the relationship between our 2 experimental groups. The red circle (right) represents the 867 transcripts found to be statistically significant (corrected $P<.05$ ) and differentially expressed at least 2-fold at 6 hours after surgical intervention in a group of mice subjected to surgery alone (group AS). The blue circle (left) represents the 216 transcripts found to be statistically significant (corrected $P<.05$ ) and differentially expressed at least 2 -fold at 6 hours after surgical intervention in a group of mice subjected to surgical intervention but given perioperative administration of the COX-2 inhibitor celecoxib (group ACS). This analysis revealed 3 distinct sets of genes: those that are differentially expressed only in response to surgical intervention alone (732 transcripts), those that are differentially expressed in response to both surgical intervention and celecoxib treatment (135 transcripts), and those that, by inference, are differentially expressed in response to celecoxib treatment alone ( 81 transcripts).

to more precisely demonstrate the relationship among the 3 experimental groups (Figure 4). This tool groups genes, experimental conditions, or both into clusters based on the similarity of their expression patterns. ${ }^{16}$ Using this approach, we demonstrated that the gene expression pattern of the celecoxib-treated surgical intervention group (group ACS) is more similar to that of the baseline anesthesia group (group A) than that of the surgical intervention-only group (group AS). This pattern of clustering was further confirmed by means of principal component analysis (data not shown).

\section{Characterization of the Inflammatory Response Initiated by Surgical Intervention Alone (Comparison of Group AS With Group A)}

Because our prime directive was the inflammatory response, we focused our analysis on known inflammatory transcripts. Of the approximately 200 known inflammatory transcripts found to be differentially expressed at 6 hours after surgical intervention, 10 chemokine and 5 chemokine receptor genes, as well as 7 interleukin receptor genes, were identified. Among commonly studied cytokines, only the gene encoding interferon $\gamma$ (IFN- $\gamma$ ) was differentially expressed at the 6-hour time point in this survey. It should be noted that most of the genes identified in these experiments could not be appropriately addressed in this treatise because of space limitations. However, a partial list of the inflammatory genes that were differentially expressed in response to surgical intervention can be found in Table 1 .

\section{Effect of Perioperative Celecoxib Administration on Surgical Intervention (Comparison of Group ACS With Group A)}

Only about 50 of the approximately 200 known inflammatory transcripts were differentially expressed in the group that received a regimen of celecoxib during the perioperative period compared with the baseline group, indicating that celecoxib successfully mitigated much of the inflammatory response after surgical intervention. The biggest difference in expression was seen in the chemokine $C \mathrm{cl} 25$, in which a transcript that was 39.4-fold increased in response to surgical intervention (group AS) was 2.5-fold decreased with the addition of perioperatively administered celecoxib (group ACS).

\section{DISCUSSION}

As proof of concept for a similar study attempting to characterize the systemic effects of surgical intervention at the whole-genome level in human subjects, we performed a pilot study comparing the gene expression profile of a group of mice subjected to anesthesia (group A) with that of a group of mice subjected to anesthesia followed by a surgical procedure (group AS). We assessed gene expression at 6 hours after surgical intervention, a time point chosen to reflect the early systemic response to surgical injury. By using animals treated with anesthesia only as our baseline group, we aimed to detect only those changes that are due to the surgical procedure itself. Furthermore, we attempted to understand the potential of the COX-2 inhibitor celecoxib to modulate this systemic response at the genomic level.

\section{Characterization of the Inflammatory Response Initiated by Surgical Intervention Alone (Group AS)}

Our survey identified 867 transcripts ( 770 genes) that displayed statistically significant differences in the surgical intervention group (corrected $P<.05$ ) and were at least 2 -fold differentially expressed. Most of the transcripts $(88.7 \%)$ showed a dramatic increase in expression in response to the surgical procedure; however, some $(11.3 \%)$ were shown to have decreases in expression (Figure 2, A). Our analyses detected 15 chemokine-related genes (both chemokines and their receptors), suggesting that they are important mediators of the surgical stress response. Chemokines are a superfamily of proinflammatory polypeptide cytokines that selectively attract and activate different cell types (ie, chemotaxis) in response to coordination by IFN- $\gamma$. Despite the importance of chemokines in immune cell recruitment, there have been no studies to our knowledge on the temporal expression of chemokines after surgical trauma. The 2 most upregulated genes in our survey, the chemokines $\mathrm{Ccl} 21$ and $\mathrm{Ccl} 25$, are potent 


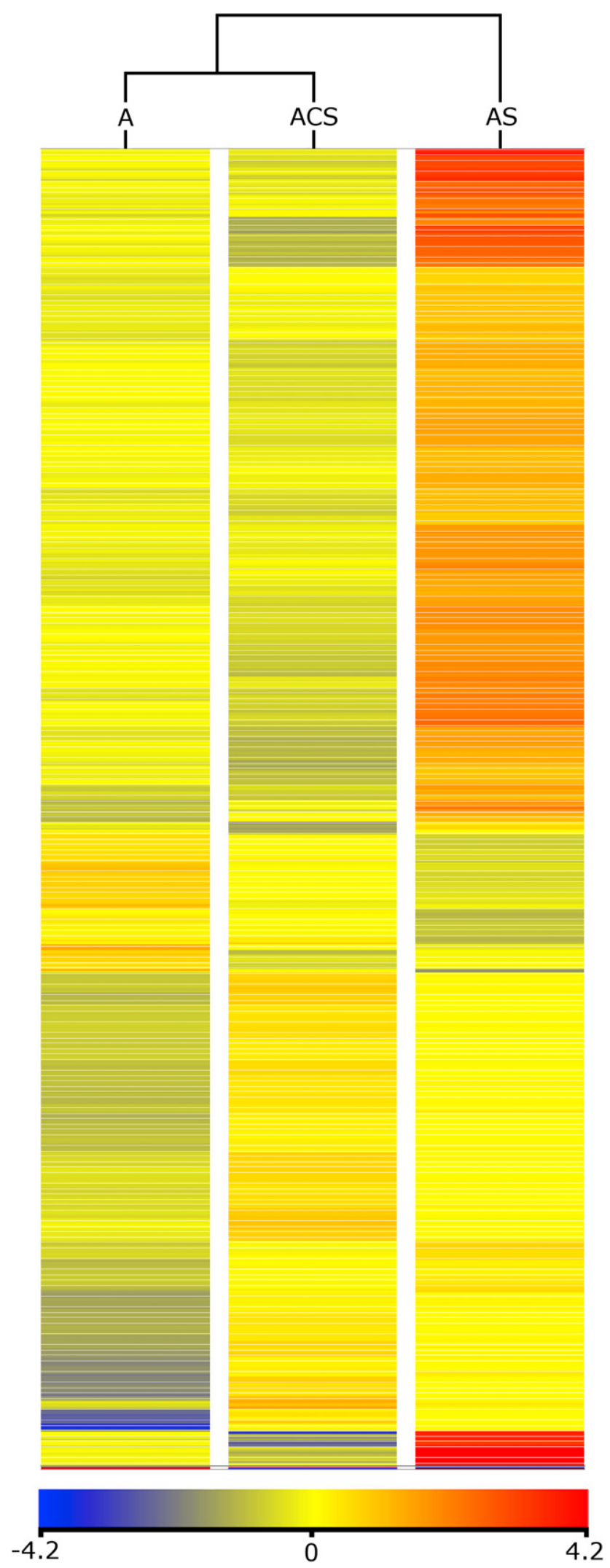

FIGURE 4. A heat map representing the 2-dimensional unsupervised hierarchical clustering that was performed on 948 differentially expressed tran- chemotactic agents for $\mathrm{T}$ cells but have also been shown to have antiapoptotic activity ${ }^{17}$ and have been implicated as promoters of metastasis and cancer progression, as have CXCL12 and CXCL13. ${ }^{18}$

Interestingly, the gene list from group AS shows significant enrichment for cell-surface receptors associated with natural killer (NK) cell-mediated cytotoxicity. Several other members of this pathway are also represented (Table 1). Further support for NK cell enrichment comes from the observation that NK cells are known to be prolific producers of and responders to chemokines ${ }^{19}$ and are both coordinated by, and producers of, the sole traditional cytokine identified in the surgical intervention group, IFN- $\gamma$. It is known that up to $80 \%$ of NK cells display CD8 antigens on their surface, and our analysis shows a significant upregulation of CD8 surface antigen, while lacking any measurable expression of CD4. It is noteworthy that both IFN- $\gamma$ and all of these killer cell lectin-like receptors are downregulated in response to surgical intervention, suggesting a diminished ability to orchestrate NK cell-mediated cytotoxicity and perhaps serving as a measure of the immunosuppression known to occur after surgical intervention.

Based on the gene expression profiles we obtained and what is known about chemokine and cytokine expression, as well as cell-type recruitment after surgical intervention, we can propose a hypothetical scenario to describe our observed results. This hypothetical paradigm asserts that the expression pattern we identified in the group undergoing surgical intervention alone (group AS) marks a time point in the acute inflammatory response at which a peripheral cell population made up of predominantly neutrophils, NK cells, or both expressing a variety of interleukin receptors on their cell surfaces are activated by cytokines produced by monocytes and macrophages at the wound site and begin expressing a selection of chemokines whose cellular activities include the recruitment of lymphocytes to the wound site, as well as immunosuppression of NK cell-mediated cytotoxicity.

Although many of the body's reactions to surgical injury are meant to facilitate the healing process, some of the inflammatory consequences can have a negative effect on cancer progression in which a compromised immune system, even for short periods, could have a significant deleterious effect. At the time of surgical intervention, anesthetic and surgically induced immune lethargy ${ }^{10}$ could allow for

scripts identified in this investigation. This tool groups genes, experimental conditions, or both into clusters based on the similarity of their expression patterns. Using this approach, we demonstrated that the gene expression pattern of the celecoxib-treated surgery group (group ACS) is more similar to that of the baseline anesthesia group (group A) than that of the surgical intervention-only group (group $A S$ ). However, unique gene expression profiles can be clearly delineated for each group. 
TABLE 1. A partial list of differentially expressed inflammatory genes

\begin{tabular}{|c|c|c|c|c|c|}
\hline $\begin{array}{l}\text { Gene } \\
\text { symbol }\end{array}$ & $\begin{array}{l}\text { Fold change } \\
\text { (AS group) }\end{array}$ & $\begin{array}{l}\text { Regulation } \\
\text { (group AS) }\end{array}$ & $\begin{array}{l}\text { Fold change } \\
\text { (group ACS) }\end{array}$ & $\begin{array}{c}\text { Regulation } \\
\text { (group ACS) }\end{array}$ & Description \\
\hline \multicolumn{6}{|c|}{ Differentially expressed genes: Cytokines and cytokine receptors } \\
\hline Ccl19 & 8.3 & Up & & & Mus musculus chemokine (C-C motif) ligand 19 \\
\hline Ccl2 & 2.8 & Up & & & Mus musculus chemokine (C-C motif) ligand 2 \\
\hline Ccl21c & 64.7 & Up & & & Mus musculus chemokine (C-C motif) ligand 21c (leucine) \\
\hline Ccl25 & 30.6 & Up & 2.5 & Down & Mus musculus chemokine (C-C motif) ligand 25 \\
\hline Ccl5 & 2.6 & Down & & & Mus musculus chemokine (C-C motif) ligand 5 \\
\hline Ccl8 & 7.0 & Up & & & Mus musculus chemokine (C-C motif) ligand 8 \\
\hline Ccr 2 & 2.0 & Up & 3.2 & Up & Mus musculus chemokine (C-C motif) receptor 2 \\
\hline Ccr3 & 2.6 & Down & & & Mus musculus chemokine (C-C motif) receptor 3 \\
\hline $\operatorname{Ccr} 9$ & 5.6 & Up & & & Mus musculus chemokine (C-C motif) receptor 9 \\
\hline$C x 3 x l 1$ & 7.5 & Up & & & Mus musculus chemokine (C-X3-C motif) ligand 1 \\
\hline Cxcll3 & 4.9 & Up & & & Mus musculus chemokine (C-X-C motif) ligand 10 \\
\hline Cxcll3 & 2.5 & Down & & & Mus musculus chemokine (C-X-C motif) ligand 13 \\
\hline Cxcll6 & 3.3 & Up & & & Mus musculus chemokine (C-X-C motif) ligand 16 \\
\hline Cxcr3 & 2.3 & Down & & & Mus musculus chemokine (C-X-C motif) receptor 3 \\
\hline \multicolumn{6}{|c|}{ Differentially expressed genes: Interleukin receptors } \\
\hline Illsap & & & 2.1 & Up & Mus musculus interleukin 18 receptor accessory protein \\
\hline Illf9 & 2.6 & Up & 3.6 & Up & Mus musculus interleukin 1 family, member 9 \\
\hline Illrl & 3.1 & Up & & & Mus musculus interleukin 1 receptor, type I \\
\hline Ill $r 2$ & 5.7 & Up & 7.3 & Up & Mus musculus interleukin 1 receptor, type II \\
\hline Illrn & 2.2 & Up & 3.2 & Up & Mus musculus interleukin 1 receptor, antagonist \\
\hline Il28ra & 7.8 & Up & & & Mus musculus interleukin 28 receptor, $\alpha$ \\
\hline$I l 2 r b$ & 2.2 & Down & & & Mus musculus interleukin 2 receptor, $\beta$ chain \\
\hline$I l 8 r b$ & 2.8 & Up & 3.5 & Up & Mus musculus interleukin 8 receptor, $\beta$ \\
\hline \multicolumn{6}{|c|}{ Differentially expressed genes associated with natural killer cells } \\
\hline Ifng & 3.1 & Down & & & Mus musculus interferon $\gamma$ \\
\hline Klra3 & 2.2 & Down & & & Mus musculus killer cell lectin-like receptor, subfamily A, member 3 \\
\hline Klra8 & 2.7 & Down & & & Mus musculus killer cell lectin-like receptor, subfamily A, member 8 \\
\hline Klra9 & 2.2 & Down & & & Mus musculus killer cell lectin-like receptor, subfamily A, member 9 \\
\hline Klrblc & 2.6 & Down & & & Mus musculus killer cell lectin-like receptor, subfamily B, member 1C \\
\hline Klrc2 & 2.7 & Down & & & Mus musculus killer cell lectin-like receptor, subfamily $\mathrm{C}$, member 2 \\
\hline Klrdl & 2.2 & Down & & & Mus musculus killer cell lectin-like receptor, subfamily $\mathrm{D}$, member 1 \\
\hline Klrkl & 3.7 & Down & & & Mus musculus killer cell lectin-like receptor, subfamily K, member 1 \\
\hline Fasi & 2.8 & Down & & & Mus musculus Fas ligand (TNF superfamily, member 6) \\
\hline Sh2dla & 2.3 & Up & & & Mus musculus $\mathrm{SH} 2$ domain protein $1 \mathrm{~A}$ \\
\hline \multicolumn{6}{|c|}{ Differentially expressed genes associated with macrophages and dendritic cells } \\
\hline$I l l b$ & & & 3.1 & Up & Mus musculus interleukin $1 \beta$ \\
\hline Chi3l3 & & & 5.5 & Up & Mus musculus chitinase 3-like 3 \\
\hline Lstl & & & 2.0 & Up & Mus musculus leukocyte-specific transcript 1 \\
\hline Ctsc & & & 2.1 & Up & Mus musculus cathepsin C \\
\hline Mpeg1 & & & 2.1 & Up & Mus musculus macrophage-expressed gene 1 \\
\hline Plscrl & 2.1 & Up & 2.1 & Up & Mus musculus phospholipid scramblase 1 \\
\hline Rab11fipl & & & 2.4 & Up & Mus musculus RAB11 family-interacting protein (class I) \\
\hline S100a6 & & & 2.1 & Up & Mus musculus S100 calcium-binding protein A6 (calcyclin) \\
\hline Sirpa & & & 2.0 & Up & Mus musculus signal-regulator protein $\alpha$ \\
\hline Mmp9 & 2.2 & Up & 2.7 & Up & Mus musculus matrix metallopeptidase 9 \\
\hline Clec4a2 & & & 2.6 & Up & Mus musculus $\mathrm{C}$-type lectin domain family 4 , member a2 \\
\hline Clec4a3 & & & 2.3 & Up & Mus musculus $\mathrm{C}$-type lectin domain family 4 , member a3 \\
\hline Clecte & & & 3.2 & Up & Mus musculus C-type lectin domain family 4 , member e \\
\hline Clec $7 a$ & & & 2.4 & Up & Mus musculus C-type lectin domain family 7, member a \\
\hline
\end{tabular}

circulating tumor cell escape and metastatic seeding, as well as providing a growth-promoting microenvironment of any quiescent micrometastases already present. Because
COX-2 inhibitors have the potential for modulating the negative effects of the systemic response to surgical intervention through their antiangiogenic, proapoptotic, and 
antimetastatic properties, we postulated that perioperative administration of these compounds could mitigate some of the increases in gene expression observed after surgical intervention. To this end, we compared the gene expression profile of our mice subjected to anesthesia (group A) with that of a group of mice subjected to anesthesia followed by our same surgical procedure but also receiving a regimen of the US Food and Drug Administration-approved COX-2 inhibitor celecoxib around the time of surgical intervention (group ACS).

\section{Effect of Perioperative Celecoxib Administration (Group ACS)}

Perioperative administration of celecoxib for 3 days before surgical intervention had a profound effect on the expression of the 867 transcripts that were shown to be differentially expressed in response to surgical intervention (Figure 2,B). The vast majority of transcripts upregulated in response to surgical intervention (group AS) were maintained near baseline levels in the celecoxib-treated group (group ACS). The largest change was seen in the chemokine $C c l 25$, which was 39.4-fold upregulated in response to surgical intervention and 2.5-fold downregulated with the addition of celecoxib. All the chemokines and all but one $(\mathrm{Ccr} 2)$ of their receptors were significantly downregulated as a result of celecoxib treatment. Given that chemokine expression of the sort seen in the surgical intervention group has frequently been linked to metastasis and cancer recurrence, ${ }^{18,20}$ this downregulation of chemokines could represent a potential mechanism for the COX-2-independent antitumor effects of celecoxib that we previously observed. ${ }^{21}$ Thus, this investigation supports the hypothesis that perioperative modulation of the surgical stress response is possible with this agent and provides the foundation for further study in this area.

Based on the Venn diagram analysis (Figure 3), only 81 transcripts can be inferred to be differentially expressed in the celecoxib-treated group outside of the context of surgical intervention. Although unknown variables could have some effect, it is reasonable to assume that the changes observed in these 81 transcripts are primarily the result of the drug treatment. Most of these 81 genes are related to the inflammatory response. Most notable among them is $I l l b$, an important cytokine in the inflammatory response that is produced by activated macrophages. It stimulates thymocyte proliferation by inducing IL-2 release, B-cell maturation and proliferation, and fibroblast growth factor activity, all important agents of immune integrity. Although not addressed in the current study, the identification of these 81 transcripts suggests that the effect of celecoxib on gene expression is not limited to modulation of the surgical stress response, and our laboratory is actively investigating these additional consequences.

Other interesting genes include mediators of dendritic cell differentiation, maturation, or both (Clec4a and Lst $)$; enhancers of cytokine production in macrophages and dendritic cells (Clec7a, Plscrl, and Tirap); macrophage markers (Ctsc and Mpegl); mediators of phagocytosis (Rabllfipl, S100A6, and Sirpa); and Chi3l3, a secreted protein that is produced primarily by macrophages during inflammation. ${ }^{22}$ Interaction between Chi3l3 and Mmp9, which is also expressed in the celecoxib-treated group, has been shown to facilitate clearance of inflammatory cells in lung tissue through a mechanism of $M m p 9$-dependent cleavagemediated modulation of Chi3l3's chemotactic activity. ${ }^{23}$ Although it is necessary to further investigate the significance of these findings, it is attractive to speculate that the dramatic anti-inflammatory effect observed in the peripheral blood of the celecoxib-treated group can be attributed to this mechanism.

Perhaps most interesting is what is no longer expressed after celecoxib treatment (group ACS). The proposed immunosuppression characterized by downregulation of the killer cell lectin-like receptors was mitigated by celecoxib treatment, as was the considerable chemokine response that has been implicated in metastasis and cancer progression. Additionally, it is quite fascinating that although the gene list from the surgical intervention-only group (group AS) implies a cell population composed of predominantly NK cells, the gene list from the celecoxib-treated surgical intervention group (group ACS) appears to indicate a cell population more characteristic of dendritic cells and macrophages.

Although the significance of these results remains complex, we can propose a hypothetical scenario to account for our observed results. This paradigm contends that celecoxib treatment modulates some of the negative effect of surgical intervention by modifying gene expression, possibly mediating surgical intervention-induced immunosuppression (as suggested by the changes induced in the killer cell lectin-like receptors), and/or mediating the expression of protumorigenic genes (eg, chemokines). Additionally, celecoxib treatment appears to result in a shift of predominant peripheral cell type from neutrophils, NK cells, or both to monocyte/macrophages, dendritic cells, or both, at least at the 6-hour time point. This shift is evidenced by the replacement of IFN- $\gamma$ with interleukin 1B, increased expression of a variety of macrophage and dendritic cell support genes, and an increase in the expression of genes involved in phagocytotic gene function. Based on a timeline of cell-type recruitment whereby neutrophils are the major leukocyte subpopulation immediately after surgical intervention, ${ }^{24}$ followed by an increase in monocyte and macrophage numbers, ${ }^{25}$ this proposed shift might also represent an overall increase in the rate at which the surgical stress response resolves, shortening the time in which a patient is exposed to the detrimental effects of perioperative immune suppression.

This study is limited by our assessment of genomic profiles at a single time point after surgical intervention. 
Obviously, the response to surgical intervention is represented by a continuum of differential gene expression, which would require a more in-depth analysis using multiple time points. Furthermore, we have attempted to study the systemic response by means of mRNA profiling of peripheral blood, which does not take into account proteins already expressed/secreted by local inflammatory cells, circulating cells, or both. It should also be noted that mRNA from whole-blood preparations can be affected by variation in leukocyte populations. Despite this limitation, the use of whole blood for assessment of gene expression has been validated in hundreds of studies. Additionally, we did not directly measure the effect of celecoxib in this study (ie, there was no celecoxib-only group), necessitating reliance on inference in our interpretation of the effects of celecoxib outside of the context of surgical intervention. This, however, was not the focus of this investigation and will be addressed in subsequent studies. Lastly, the experiment was performed on young mice. Although older mice might have a different immune response than younger mice, all our experiments were performed on mice of similar ages, and therefore the response to celecoxib is still valid. While recognizing these limitations, we believe that this research represents a valuable contribution to the overall understanding of the effect of surgical wounding and its role in cancer progression.

In summary, our data show that there is a remarkable differential gene expression induced by surgical intervention, as evidenced by whole genomic profiling of peripheral blood 6 hours after a surgical injury. Additionally, the perioperative administration of the COX-2 inhibitor celecoxib profoundly affects this response. This is the first attempt, to our knowledge, to investigate these phenomena at the genomic level. Although in-depth analysis of all involved genes and pathways is beyond the scope of this article, we believe that many of the potentially deleterious effects of the surgical stress response that have been shown to promote tumorigenesis can be mitigated through the use of perioperative celecoxib treatment. Although ongoing animal and human studies are currently being carried out in our laboratory to fully evaluate the consequences of pharmacologic intervention of the surgical stress response, the preliminary data presented here appear to support its potential use.

\section{References}

1. Pape HC, Tsukamoto T, Kobbe P, Tarkin I, Katsoulis S, Peitzman A. Assessment of the clinical course with inflammatory parameters. Injury. 2007;38:1358-64.

2. Chyczewska E, Mroz RM. Cytokines in lung cancer. Rocz Akad Med Bialymst. 1997;42(suppl 1):8-22.
3. Craig SR, Leaver HA, Yap PL, Pugh GC, Walker WS. Acute phase responses following minimal access and conventional thoracic surgery. Eur J Cardiothorac Surg. 2001;20:455-63.

4. Franke A, Lante W, Fackeldey V, Becker HP, Kurig E, Zoller LG, et al. Proinflammatory cytokines after different kinds of cardio-thoracic surgical procedures: is what we see what we know? Eur J Cardiothorac Surg. 2005;28:569-75.

5. Shiromizu A, Suematsu T, Yamaguchi K, Shiraishi N, Adachi Y, Kitano S. Effect of laparotomy and laparoscopy on the establishment of lung metastasis in a murine model. Surgery. 2000;128:799-805.

6. Zimmermann AK, Simon P, Seeburger J, Hoffmann J, Ziemer G, Aebert H, et al. Cytokine gene expression in monocytes of patients undergoing cardiopulmonary bypass surgery evaluated by real-time PCR. J Cell Mol Med. 2003;7:146-56.

7. Seeburger J, Hoffmann J, Wendel HP, Ziemer G, Aebert H. Gene expression changes in leukocytes during cardiopulmonary bypass are dependent on circuit coating. Circulation. 2005;112(suppl):I224-8

8. Tsuchiya Y, Sawada S, Yoshioka I, Ohashi Y, Matsuo M, Harimaya Y, et al. Increased surgical stress promotes tumor metastasis. Surgery. 2003;133:547-55.

9. Carvalho B, Clark DJ, Angst MS. Local and systemic release of cytokines, nerve growth factor, prostaglandin E2, and substance $\mathrm{P}$ in incisional wounds and serum following cesarean delivery. J Pain. 2008;9:650-7.

10. Kurosawa S, Kato M. Anesthetics, immune cells, and immune responses. J Anesth. 2008;22:263-77.

11. Hirai T, Yoshimoto A, Iwata T, Yamashita Y, Kuwahara M, Toge T. Enhancing effect of thoraco-laparotomy on liver metastasis and the role played by active oxygen in its mechanism. Surg Today. 1997;27:1040-5.

12. Diperna CA, Bart RD, Sievers EM, Ma Y, Starnes VA, Bremner RM. Cyclooxygenase-2 inhibition decreases primary and metastatic tumor burden in a murine model of orthotopic lung adenocarcinoma. J Thorac Cardiovasc Surg. 2003;126: 1129-33.

13. Velentgas P, West W, Cannuscio CC, Watson DJ, Walker AM. Cardiovascular risk of selective cyclooxygenase- 2 inhibitors and other non-aspirin non-steroidal anti-inflammatory medications. Pharmacoepidemiol Drug Saf. 2006;15:641-52.

14. Benish M, Bartal I, Goldfarb Y, Levi B, Avraham R, Raz A, et al. Perioperative use of beta-blockers and COX-2 inhibitors may improve immune competence and reduce the risk of tumor metastasis. Ann Surg Oncol. 2008;15:2042-52.

15. Benjamini B, Hochberg Y. Controlling the false discovery rate: a practical and powerful approach to multiple testing. J R Statist Soc B. 1995;57:289-300.

16. Eisen MB, Spellman PT, Brown PO, Botstein D. Cluster analysis and display of genome-wide expression patterns. Proc Natl Acad Sci U S A. 1998;95:14863-8.

17. Youn BS, Yu KY, Oh J, Lee J, Lee TH, Broxmeyer HE. Role of the CC chemokine receptor 9/TECK interaction in apoptosis. Apoptosis. 2002;7:271-6.

18. Feng LY, Ou ZL, Wu FY, Shen ZZ, Shao ZM. Involvement of a novel chemokine decoy receptor CCX-CKR in breast cancer growth, metastasis and patient survival. Clin Cancer Res. 2009;15:2962-70.

19. Robertson MJ. Role of chemokines in the biology of natural killer cells. J Leukoc Biol. 2002;71:173-83.

20. Wang JM, Deng X, Gong W, Su S. Chemokines and their role in tumor growth and metastasis. J Immunol Methods. 1998;220:1-17.

21. Backhus LM, Petasis NA, Uddin J, Schonthal AH, Bart RD, Lin Y, et al. Dimethyl celecoxib as a novel non-cyclooxygenase 2 therapy in the treatment of non-small cell lung cancer. J Thorac Cardiovasc Surg. 2005;130:1406-12.

22. Welch JS, Escoubet-Lozach L, Sykes DB, Liddiard K, Greaves DR, Glass CK. TH2 cytokines and allergic challenge induce $\mathrm{Ym} 1$ expression in macrophages by a STAT6-dependent mechanism. J Biol Chem. 2002;277:42821-9.

23. Greenlee KJ, Corry DB, Engler DA, Matsunami RK, Tessier P, Cook RG, et al. Proteomic identification of in vivo substrates for matrix metalloproteinases 2 and 9 reveals a mechanism for resolution of inflammation. J Immunol. 2006; 177:7312-21.

24. Swank DW, Moore SB. Roles of the neutrophil and other mediators in adult respiratory distress syndrome. Mayo Clin Proc. 1989;64:1118-32.

25. Faist E, Storck M, Hultner L, Redl H, Ertel W, Walz A, et al. Functional analysis of monocyte activity through synthesis patterns of proinflammatory cytokines and neopterin in patients in surgical intensive care. Surgery. 1992;112:562-72. 


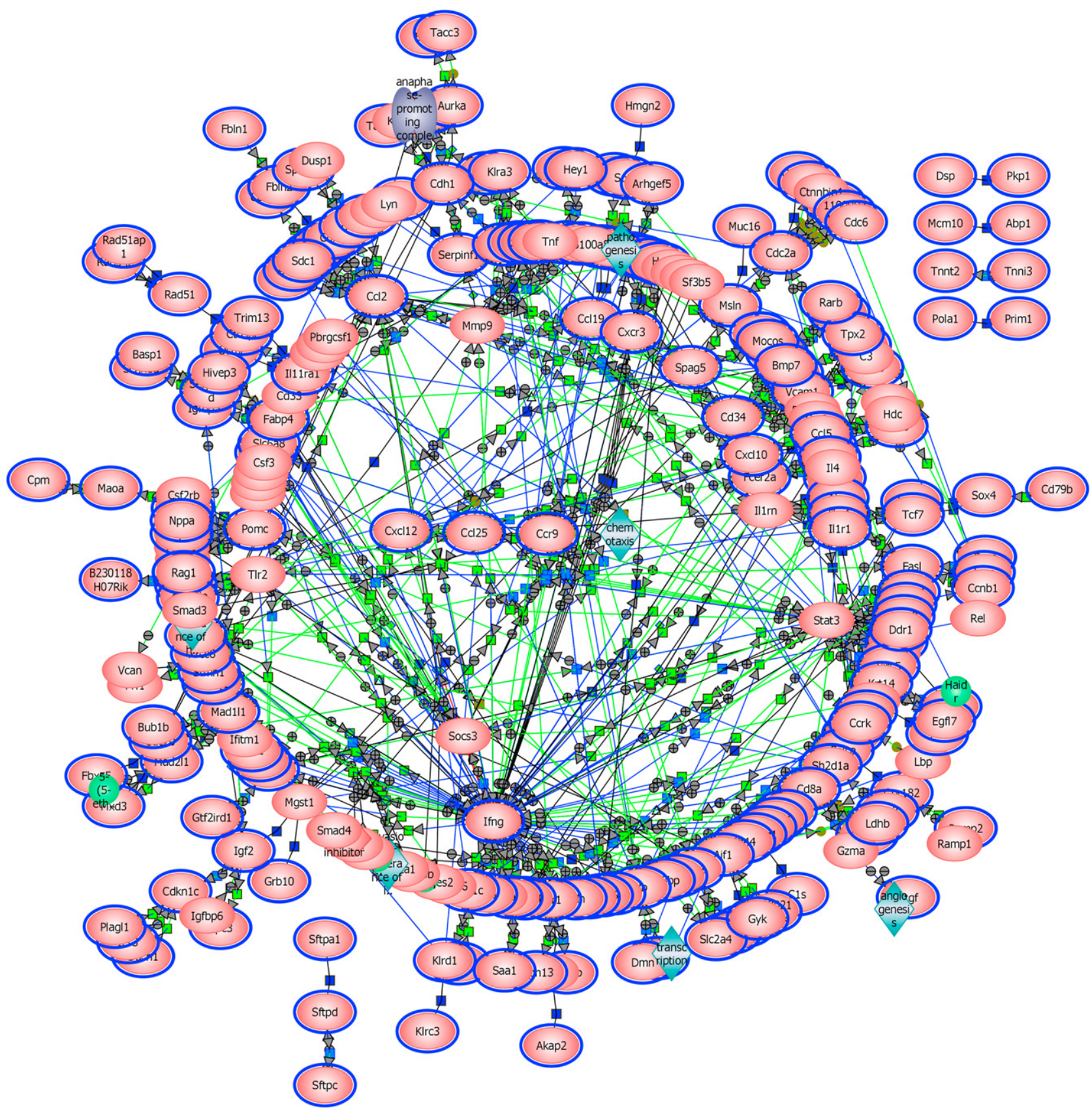

FIGURE E1. Pathway analysis was performed in both GeneSpring and through BioRag (www.biorag.org) by using the BioCarta, KEGG, and GenMAPP databases. The figure shows the results of these analyses for the surgical intervention group (group AS). Pink ovals represent proteins in the pathways, and those highlighted in blue are represented in our experimental gene list. It can be seen that the central mediator of the resulting pathways is interferon $\gamma$ (Ifng) and that the most significant pathways (displayed as blue diamonds) are transcription (connectivity 6474), angiogenesis (connectivity 2735), pathogenesis (connectivity 1639), chemotaxis (connectivity 898), evasion or tolerance of host immune response (connectivity 35), and evasion or tolerance of host immune response of other organisms during symbiotic interaction (connectivity 35). An anaphase-promoting complex (connectivity 477) can also be observed. Other significant pathways observed, although too numerous to list fully, include apoptotic signaling in response to DNA damage, antiapoptotic signaling from Igflr, $\mathrm{T}_{\mathrm{H}}$ 2-mediated cytokine gene expression, RAS signaling, G1 to $\mathrm{S}$ transition, mitogen-activated protein kinase activation, and T-cell activation. Gene Ontology (GO) is a database that assimilates all available information to categorize genes according to function. A search for GO associations within the 867 transcripts identified in the surgical intervention group revealed significant levels of GO enrichment $(P<.05)$. Not surprisingly, many of the transcripts that were differentially expressed in response to surgical intervention were associated with the surgical stress response. Other biological processes observed were cell division, cell cycle, DNA replication, development, cytoskeletal organization, and chemotaxis. It should be noted that these functional categories are not exclusive, and many transcripts belong to more than 1 category. 


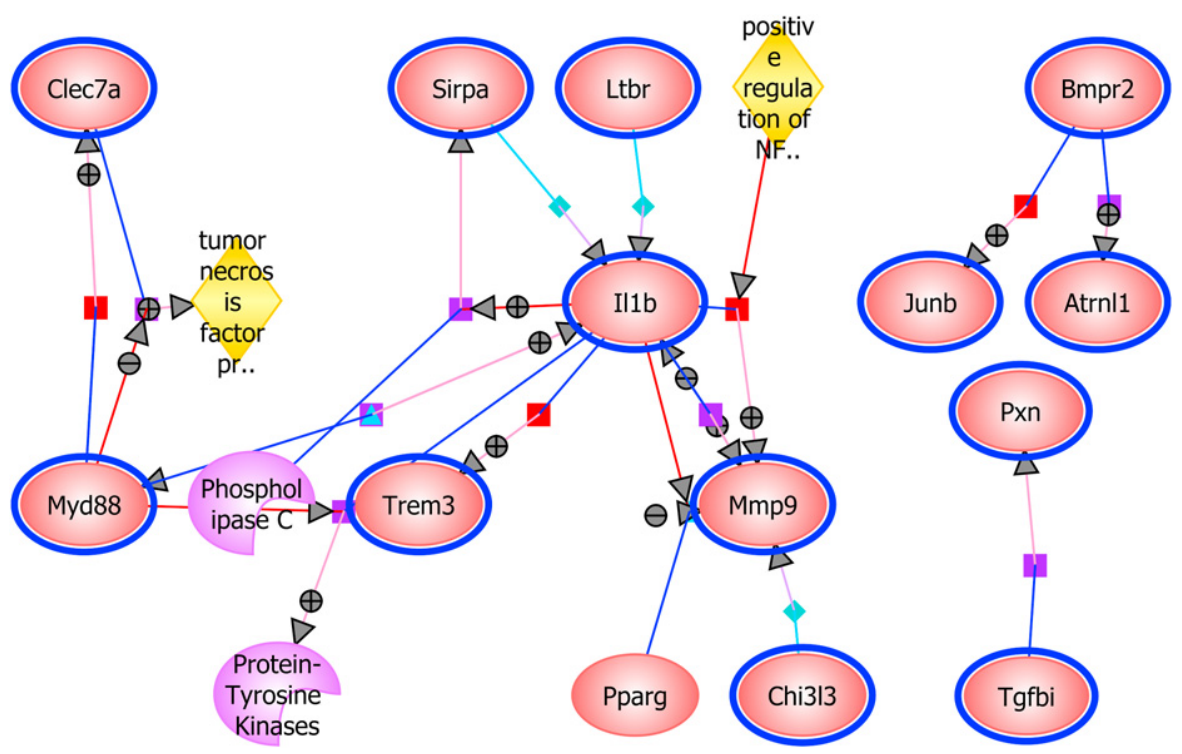

FIGURE E2. Pathway analysis was performed in both GeneSpring and with BioRag (www.biorag.org) by using the BioCarta, KEGG, and GenMAPP databases. This figure shows the results of these analyses for the celecoxib-treated surgical intervention group (group AS). Pink ovals represent proteins in the pathways, and those highlighted in blue are represented in our experimental gene list. It can be seen that the central mediator of the resulting pathways is interleukin $1 \beta(I l l b)$ and that the most significant pathways (displayed as gold diamonds) are tumor nuclear factor $(N F) \kappa \mathrm{B}$ transcription factor activity (connectivity 1438) and tumor necrosis factor production (connectivity 1079). Additionally, this gene list is associated with 2 enzymes (displayed as purple sickles): phospholipase $\mathrm{C}$ and protein tyrosine kinases. Fewer but still significant $(P<.05)$ Gene Ontology $(\mathrm{GO})$ enrichment associations were found among the 216 transcripts identified in the celecoxib-treated group, and many of the same biological processes related to the surgical stress response identified in the above comparison were represented here as well, albeit at lower frequencies. Interestingly, this group contained a biological process not found in the surgical intervention group: regulation of phagocytosis/endocytosis. Subsequent analysis revealed that this phagocytosis/endocytosis function was contained within the 81 transcripts mediated solely by celecoxib. 